\title{
Review of brain MRI image segmentation methods
}

\begin{abstract}
Brain image segmentation is one of the most important parts of clinical diagnostic tools. Brain images mostly contain noise, inhomogeneity and sometimes deviation. Therefore, accurate segmentation of brain images is a very difficult task. However, the process of accurate segmentation of these images is very important and crucial for a correct diagnosis by clinical tools. We presented a review of the methods used in brain segmentation. The review covers imaging modalities, magnetic resonance imaging and methods for noise reduction, inhomogeneity correction and segmentation. We conclude with a discussion on the trend of future research in brain segmentation.
\end{abstract}

Keyword: Brain, MRI, Segmentation 